\title{
Air pollution linked to multiple sclerosis and stroke
}

the incidence
of both
ischaemic
stroke and
haemorrhagic
stroke was
significantly
associated
with the
level of long-
term PM $_{2.5}$
exposure

gु
Extreme air pollution can increase the risk of neurological disease, suggest the results of two new studies published in the British Medical Journal and Acta Neurologica Scandinavia. The findings could inform environmental and health policy, particularly in low-income countries.

Exposure to air pollution in the form of particles with a diameter $\leq 2.5 \mu \mathrm{m}\left(\mathrm{PM}_{2.5}\right)$ has previously been associated with an increased risk of stroke. However, most of the studies that identified this association were conducted in North America and Europe, where $\mathrm{PM}_{2.5}$ concentrations are relatively low. In a new study conducted in China, Dongfeng $\mathrm{Gu}$ and colleagues aimed to provide insight into the health effects of the extreme air pollution that occurs in middle-income and low-income countries.

"Although previous studies of short-term exposure (for example, over a few days) to ambient $\mathrm{PM}_{2.5}$

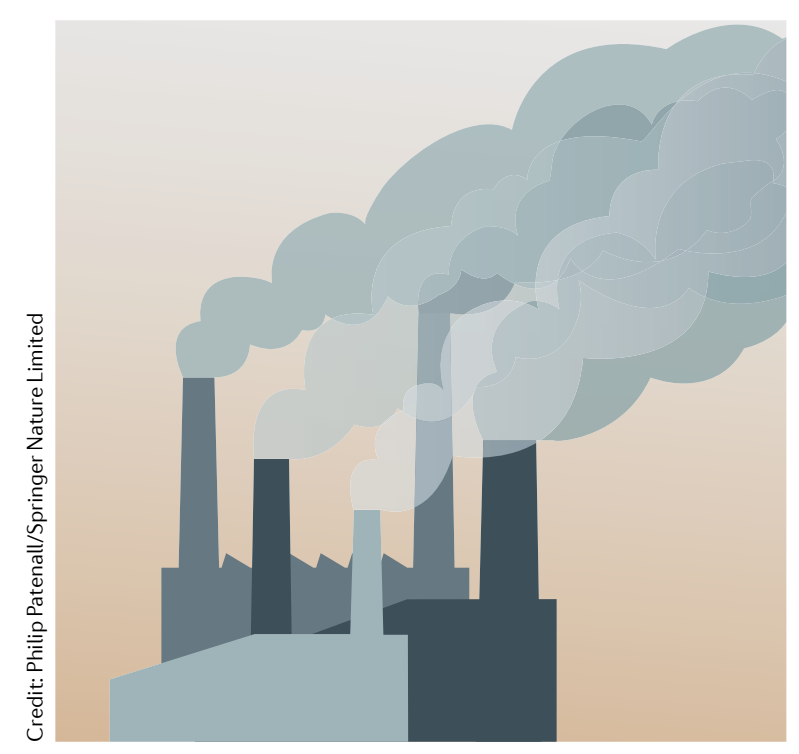

have reported increased risks of hospital admission for stroke in China, evidence of the accumulated damage on cardiovascular health caused by long-term exposure to air pollution was limited," explain the researchers in the paper.

$\mathrm{Gu}$ and colleagues calculated a time-weighted average exposure to $\mathrm{PM}_{2.5}$ for 117,575 participants enrolled in an existing prospective cohort study. To do this, they used data from NASA satellites to estimate the monthly ambient $\mathrm{PM}_{2.5}$ levels across China from 2000 to 2015 and combined these estimations with participants' home addresses to calculate their exposure to $\mathrm{PM}_{2.5}$.

Participants were enrolled in the study between 1992 and 2008 and were monitored for stroke incidence until 2012-2015. During data analysis, the researchers controlled for potential confounding factors such as age, gender, urbanization of participants' residence, smoking status, alcohol intake, education level, work-related physical activity, body mass index and hypertension.

Each $10 \mu \mathrm{g} / \mathrm{m}^{3}$ increase in long-term $\mathrm{PM}_{2.5}$ exposure was associated with a $13 \%$ increase in the incidence of stroke. In addition, the incidence of both ischaemic stroke and haemorrhagic stroke was significantly associated with the level of long-term $\mathrm{PM}_{2.5}$ exposure.

"These findings are meaningful for both environmental and health policy development related to air pollution and stroke prevention, not only in China, but also in other low-income and middle-income countries," say the researchers in the paper.

In the other new study, researchers led by Cem Bölük directly compared the incidence of multiple sclerosis (MS) in two Turkish cities with different levels of air pollution. "Although various studies of the environmental risk factors suggest a link between exposure to environmental factors and MS, there is still much that remains unknown," note the researchers in the paper.

Geographical, cultural, racial and economic profiles of the two cities were similar. However, in one city, levels of airborne pollutants exceeded WHO threshold values.

Bölük and colleagues used a questionnaire to screen inhabitants of the two cities. If a participant indicated on the questionnaire that they had experienced MS-like symptoms, they were examined by a neurologist and their previous medical records were reviewed. In total, 54,224 individuals were screened and 41 individuals were diagnosed with MS.

The age-standardized prevalence of MS was 97.4/100,000 in the city with high levels of air pollution and $47.2 / 100,000$ in the city with low levels of air pollution, indicating an association between air pollution and increased prevalence of the disease. However, the researchers point out several limitations of the work, including the small size of the study population and the inability to control for potential confounders, such as vitamin D level or history of smoking.

"The results of this study suggest that air pollution may be an aetiological factor in MS," conclude the researchers in the paper. "More case-control studies investigating environmental effects on MS are needed in order to shed more light on this area."

Sarah Lemprière

ORIGINAL ARTICLES Huang, K. et al. Long term exposure to ambient fine particulate matter and incidence of stroke: prospective cohort study from the China-PAR project. BMJ. https://doi.org/ 10.1136/bmj.l6720 (2019) | Türk Börü, Ü. et al. Air pollution, a possible risk factor for multiple sclerosis. Acta. Neurol. Scand. https://doi.org/ 10.1111/ane.13223 (2020) 